\title{
End Relative to Reference Time Point
}

National Cancer Institute

\section{Source}

National Cancer Institute. End Relative to Reference Time Point. NCI Thesaurus. Code C82558.

An indication or description that the end of the observation is before, during or after a reference moment in time. 